\title{
Design of intelligent Bicycle kit
}

\section{Qian Wang ${ }^{1, ~ a, ~ Z h e x i a n g ~ Z o u ~}{ }^{2, b}$ and Weijie Tang ${ }^{3, c}$}

\author{
${ }^{1}$ Bei Jing Institute of Technology.Zhu Hai,ZhuHai City,GuangDong Province, In China \\ ${ }^{2}$ Bei Jing Institute of Technology.Zhu Hai,ZhuHai City,GuangDong Province, In China \\ ${ }^{3}$ Bei Jing Institute of Technology.Zhu Hai,ZhuHai City,GuangDong Province,In China

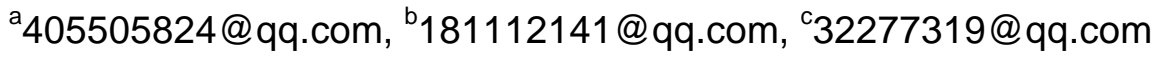

\begin{abstract}
Keywords: intelligent bicycle kit;Security remind;movement monitoring ;MCU
\end{abstract}
Abstract. Under the influence of environmental problems, there are increasingly large scale fitness group puts forward higher requirements on the bike.At present, there are various form and pecifications of the bicycle. The recombination of bicycle when unpacking the case affected the the trend of the development of the intelligent direction for the bike. To solve the similar problems, this design mainly uses the single chip microcomputer as control chip to build a bike intelligent kit, using sensor technology to achieve function of speed display, light indication lights, motion acceleration sensor monitoring, anti-theft alarm functions, to simple installation to various styles of bicycle,optimizing the user's riding experience.

\section{Introduction}

Bike as the consumer products with utilization rate and high recognition.Compared with the new energy vehicles, it has low barriers to entry and more easier to combined with the Internet.In addition, improvement of bicycle has been in a mechanical level, it didn't introduced intelligent, neither science nor technology,bicycle plus smart combination maybe have larger growth space.

The biggest advantage of this design bicycles intelligent kit is: Using wireless communications suite of bicycles don't need to buy a new smart bike, at the same time, it will be able to realize the corresponding function with no wiring, to a certain extent, save the bicycle cost of upgrading for consumers; Rely on the data of two axes collected by gyroscope to judge the bicycle on the slope of the brake action is more accurate than that rely on a shaft data;Using gyroscope module and setting a algorithm to judge the bicycle is being accidentally hit by a stranger or it is the threat of theft; With the method of wireless communications, design the mode of a host communicate with multiple auxiliary.Through the above four optimization design, we can perfect the function of the intelligent bicycle, simplify the structure of intelligent bike.

\section{Design of the intelligent bicycles kit}

Bike smart car suite design including hardware design and software development, in order to realize the state of cycling (cycling mode) of the speed display, light a lamp, acceleration induction brake lights, function of IC card to identify the owner, and parking state "anti-theft mode" anti-theft alarm and SMS alerts functions.

Circuit design. The structure of Bicycle intelligent vehicle suite is shown in Fig.1, choosing hall sensor 3144E with selection switch to check the bike speed;choosing photosensitive sensor testing environment light to achieve the control in a light;choosing NRF24L01 chip as wireless communication module, NRF24L01 chip has been adopted, we can give the owner with wireless keys;choosing MPU6050 gyroscope chip to realize security reminder and automatic testing function such as braking action; choosing ULN2803 chip to driver the LED lights before and after; choosing IC card read module to achieve security settings; choosing the GSM module to send alarm information to the public security net and the owner phone;choosing STC15W408AS single-chip microcomputer as train control chip of main control board, warning light module and display module[1]. 
Design of Software and Experiment of the Function. Master software program mainly complete three tasks: the value of $Y$ axis accelerometer with the rest two axis of gyroscope chip MPU6050 to judge bike brake action, and judge the bicycle is being accidentally hit by a stranger or the threat of theft; Through NRF24L01 chip to control tail light for wireless communications[2]. Program block diagram is shown in Fig.2.

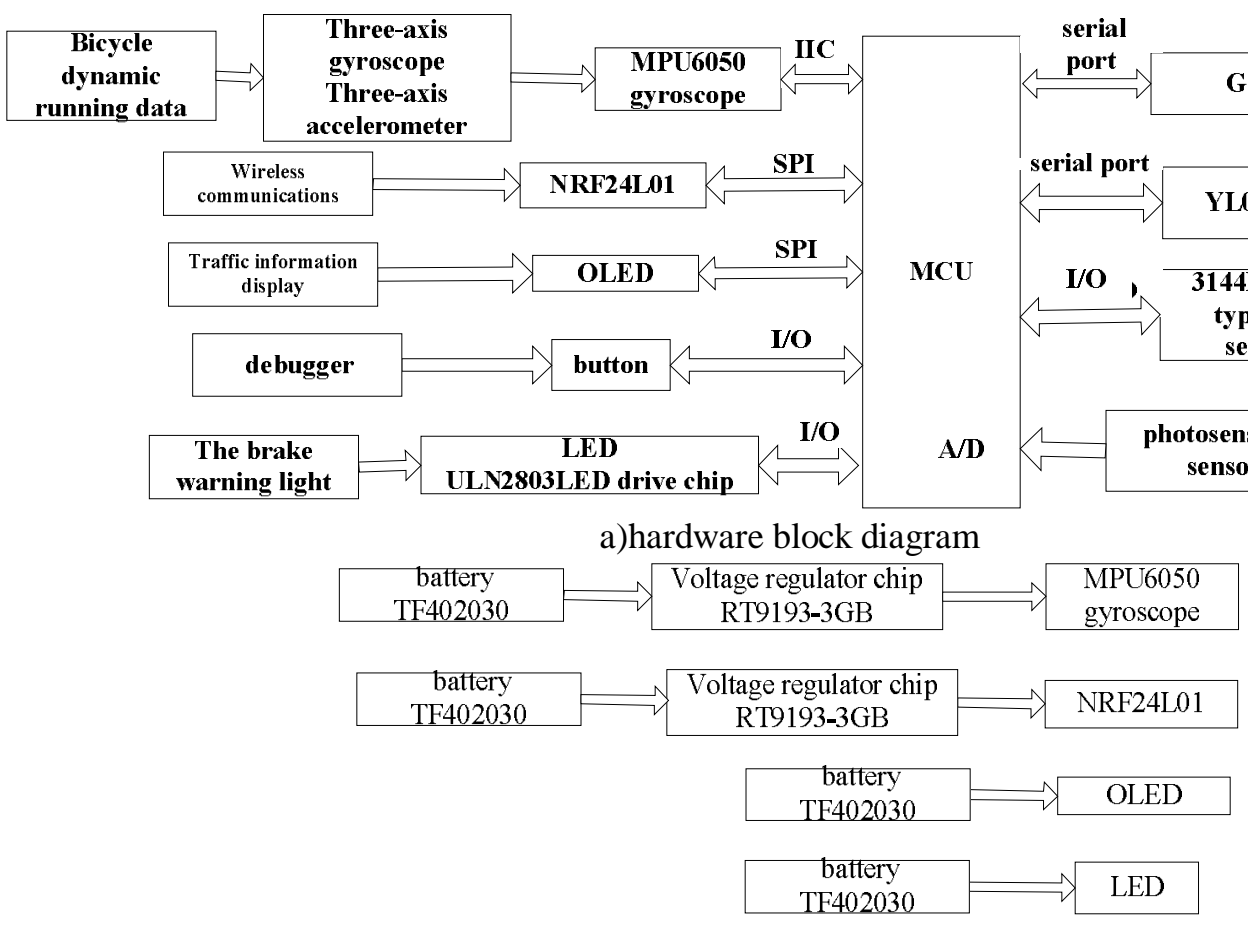

b)Power supply unit

Fig. 1. intelligent vehicle suite system block diagram

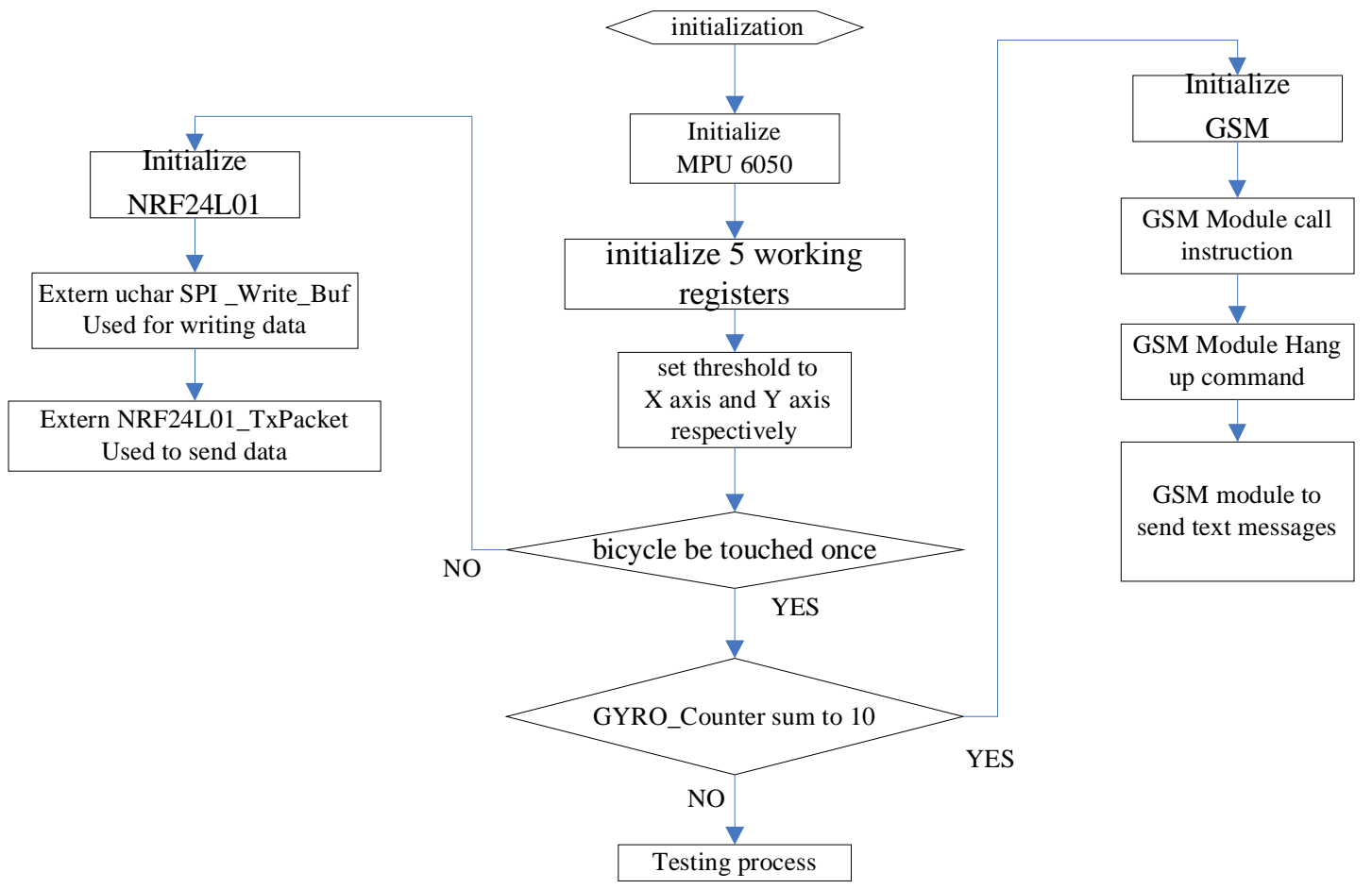

Fig. 2. main program flow chart

Design of the judgement for braking action Judgment braking action mainly through the data collected by the gyroscope to complete the analysis judgment, the main steps are as follows:first initialized all of the peripherals during power on; and then MPU6050 module and MCU communicate via the IIC communication,such as we need to read high 8 bits of data of $\mathrm{X}$ axis 
accelerometer, can be written as:num=Single_ReadI2C(ACCEL_XOUT_H);Finally analysising the data, in order to be more intuitive to observe the change trend of the data,it can display real-time change of numerical with virtual oscilloscope, the display effect is shown in Fig.3,Abscissa for time, ordinate for value[3].

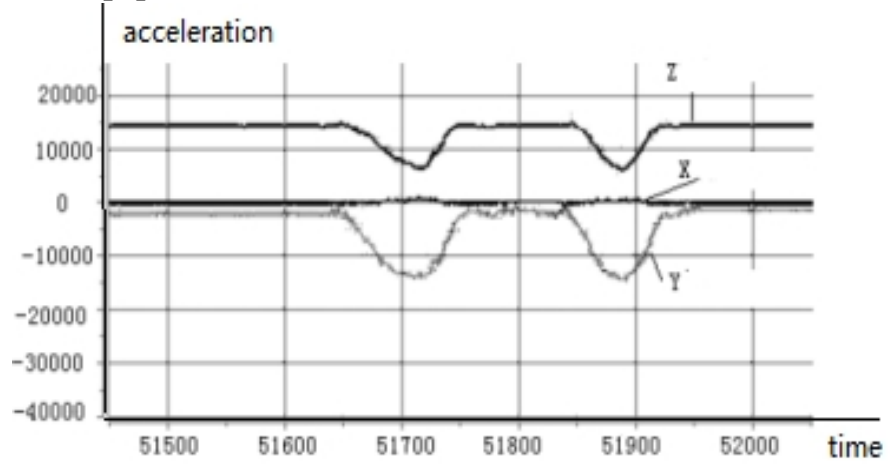

Fig.3. The virtual oscilloscope display effect graph

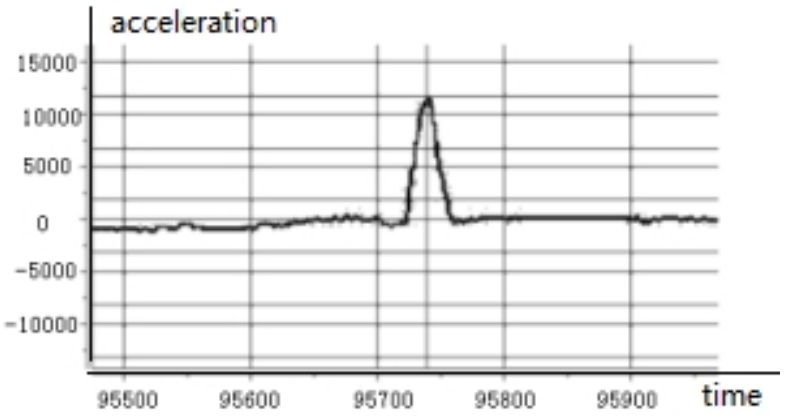

Fig.4. Y axis accelerometer of Heavy braking graph

When designing judgment for braking action, we only need to use the value of $Y$ axis accelerometer, the rest of the two axis can be used as the auxiliary judgment. We measured the data of three axis as a reference during a constant speed, and then we got the curve of the $Y$ axis by road test and heavy brake. The data measured by the Y-axis acceleration when heavy braking is shown in Fig.4,Reached a peak of $11384 \mathrm{~m} / \mathrm{min}^{2}$. The $0-11384$ can be divided into eight interval, representing different braking power.

Trigger Design of Burglar Alarm. When designing alarms trigger condition algorithm, it is necessary to use the value of the $\mathrm{X}$ axis and $\mathrm{Y}$ axis of gyroscopecontrol. When bicycle was touched at the moment, each data for the axis of gyroscope have great changes, which is shown in Fig.5.

Setting two threshold values for $\mathrm{X}$ axis and $\mathrm{Y}$ axis , respectively, when the absolute value of an arbitrary axis of gyroscope value is greater than the corresponding threshold value, whitch can be judged that the bicycle be touched once,GYRO_Counter plus once,Considering the accidentally touch the bike, according to the practical test, when variable GYRO_Counter sum to 10 ,GYRO Counter will trigger the alarm, meanwhile GYRO_Counter variable will be reset.

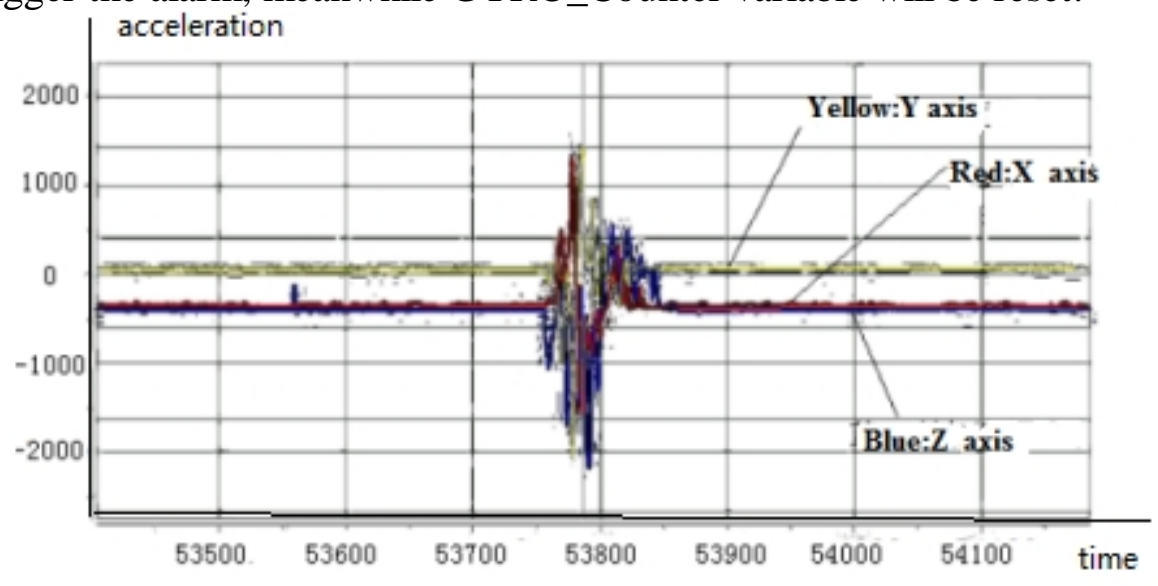

Fig.5.Touch simulation gyroscope graph

Wireless Communication. Pay attention to two aspects in the wireless communication module. (1) to ensure the receiving end including traffic warning taillight module and display module can receive data at the same time; (2) main control panel can stop NRF24L01 chip send instructions in order to achieve the purpose of reducing power consumption in anti-theft mode.

\section{Integrated System Test}

Debug Module GSM. A serial port debug tool to receive buffer receives the single chip microcomputer instruction is a text mode, As confirmation of single-chip microcomputer serial port 
send commands are HEX files, it can be connected with GSM module to make calls, etc.after it detected correctly[4].

Calibration of Gyroscope. Signal waveform of $\mathrm{X}$ axis and $\mathrm{Y}$ axis acceleration gyroscope measured by digital oscilloscope is shown in Fig.6, when the bike in uniform motion.Multiple measurement data and then calculate them can get the $X$ axis acceleration averages value, $+452 \mathrm{~m} / \mathrm{min}^{2} \mathrm{Y}$ axis Average acceleration is $+1220 \mathrm{~m} / \mathrm{min}^{2}$, In addition of $\mathrm{X}$ axis and $\mathrm{Y}$ axis direction and set in the opposite direction,After calculate the average in the program on the available data compensation and the correction of waveform diagram is shown in Fig.7.

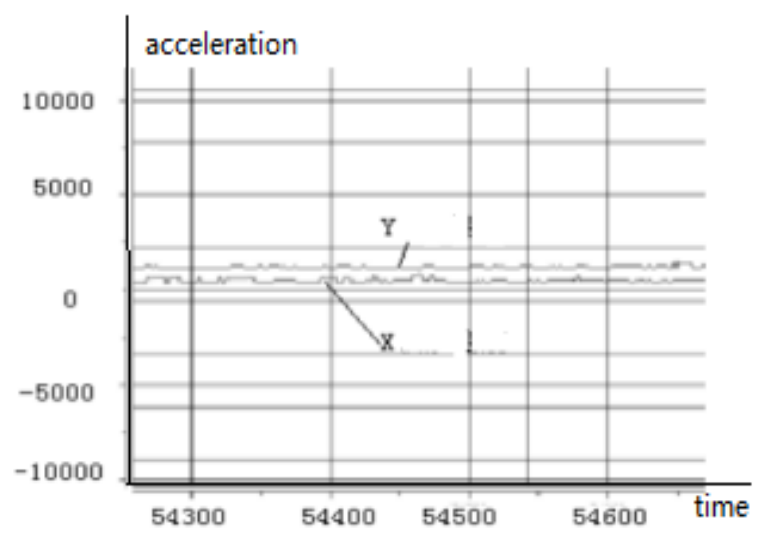

Fig.6. Before calibration waveform

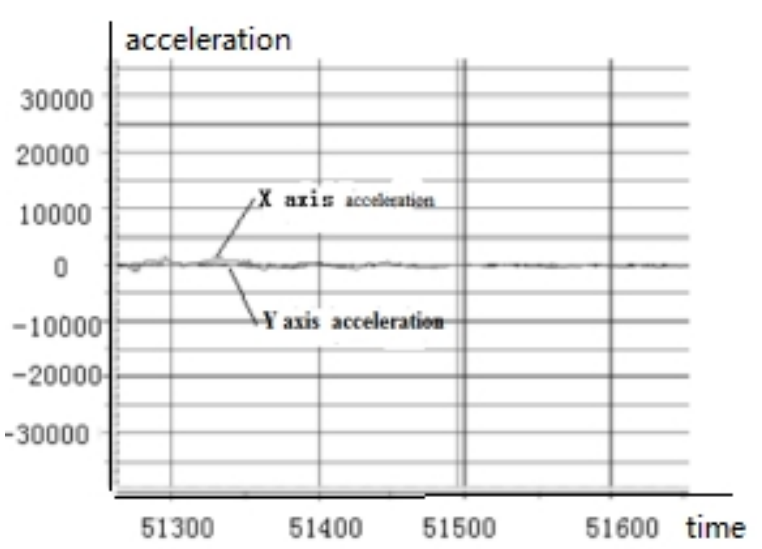

Fig.7. After calibration waveform

The problem of NRF24L01 transmission int data type . When trying to use NRF24L01 wireless module transfer 3 axis acceleration data to the driving display module, found a gyroscope random numerical change, very confused.Then detect the sending is defined as an arrayuchar TxBuf[16]; uchar's the numerical range is $0-255$,Value of the gyroscope is defined as type int,Numerical range is -65536 65536, Obviously one of the elements in the array TxBuf are not fully loaded a acceleration data.So you need to put a shaft acceleration value broken down into three elements in the array TxBuf.And a symbol used to distinguish between positive and negative, these elements and then sent to the road after the splitting of display module, equivalent to send data compiled again. X Y Z axis acceleration value of master control board will be sent to driving display module and successfully displayed during debugging.

Debugging Induction Brake Lights . According to $\mathrm{Y}$ axis acceleration road test data,the four times inertial sliding experimental $\mathrm{Y}$ axis data is shown in table 1, the average calculated for $-388.25 \mathrm{~m} / \mathrm{min}^{2}, \mathrm{Y}$ axis data of four heavy braking experiment is shown in table 2, the average calculated for $9081 \mathrm{~m} / \mathrm{min}^{2}$.To conclude the two values set for the threshold of the programe.Y axis acceleration measurement value sent to the warning light module can realize the function of the display that LED taillight adjust brightness with brake power.

Table 1. Y axis acceleration peak and average of inertia Sliding experiment ( Unit : $\mathrm{m} / \mathrm{min}^{2}$ )

\begin{tabular}{|c|c|c|c|c|c|}
\hline & Sample.1 & Sample.2 & Sample.3 & Sample.4 & Average \\
\hline Y axis acceleration & -398 & -372 & -381 & -402 & -388.25 \\
\hline
\end{tabular}

Table 2. Y axis acceleration peak and average of Heavy braking experimental (Unit : $\mathrm{m} / \mathrm{min}^{2)}$

\begin{tabular}{|c|c|c|c|c|c|}
\hline & Sample.1 & Sample.2 & Sample.3 & Sample.4 & Average \\
\hline Y axis acceleration & 9014 & 8984 & 9201 & 9125 & 9081 \\
\hline
\end{tabular}

Security Alerts Debugging .Simulation bicycle is stolen, the measured $\mathrm{X}$ axis and $\mathrm{Y}$ axis curve of gyroscope, the characterized of which appears peak for many times, is shown in Fig.8, take four peak value which is shown in table 3, in the burglar triggered judgment, the $\mathrm{Y}$ axis of data of gyroscope as the basis of the judgement. Y axis numerical values chages between $239 \mathrm{~m} / \mathrm{min}^{2}$ and $542 \mathrm{~m} / \mathrm{min}^{2}$, So you can take the middle value as a threshold, When the $\mathrm{Y}$ axis gyro value across the threshold, it is judged to be triggered a theft. 


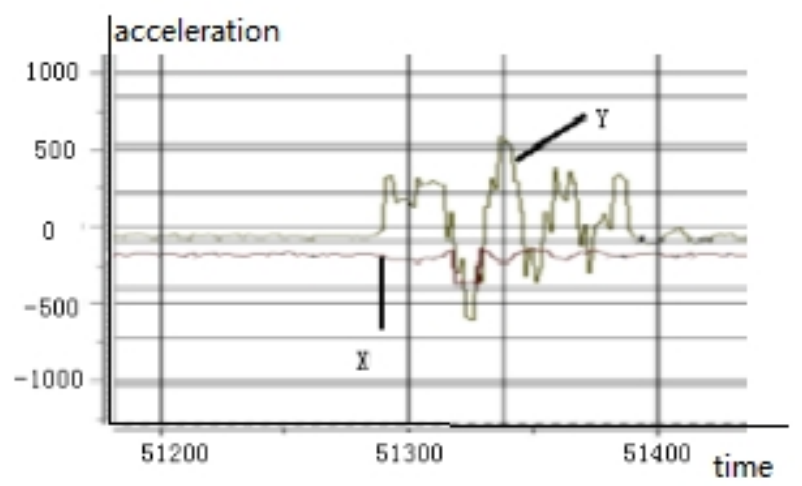

Fig.8. Simulation Diagram

Table 3. X Y axes numerical samples under Touch simulation ( Unit : $\mathrm{m} / \mathrm{min}^{2}$ )

\begin{tabular}{|c|c|c|c|c|}
\hline & Sample.1 & Sample.2 & Sample.3 & Sample.4 \\
\hline $\mathrm{X}$ axis & -215 & -231 & -211 & -182 \\
\hline Y axis & 239 & 542 & 521 & 292 \\
\hline
\end{tabular}

\section{Conclusions}

Bike smart car kit has huge development potential and broad prospects for development, the design on the basis of predecessors, extended the function of the following : 1. Bicycle speed function,the design instead of a magnet after measured the speed ,the speed display effect is relatively satisfied;2.photosensitive indicating light:the final debugging results suited by sensing the environment light to choose to open photosensitive indicating light, and it will automatically shut down in a good place with Good light in the night.3. When the gyroscope detect a braking action, it will lights up the different number of brake leds ; 4.Anti-theft function of SMS alerts, actual testing capabilities can most accurate .So this design enables user's riding experience more intelligent, more rich and colorful.

\section{References}

[1] V. Yu. Teplov,A. V. Anisimov. Thermostatting System Using a Single-Chip Microcomputer and Thermoelectric Modules Based on the Peltier Effect[J] ,2002.

[2] D.Tulone.A resource-efficient time estmation for wireless sensor networks.In Proc.of the 4th Workshop of Principles of mobile Computing,pp.52-59,Oct 2004.

[3] George Lee,Karina $\mathrm{Ng}$,Edmond Kwang.Design of ring oscillator based voltage controlled oscillator. Project Final Report[R]. 2005

[4] MeeHan Joanne,Muir Lindesy.SCM in Merseyside SMEs:Benefits and barriers[J].TQM Journal.2008 